\title{
DESARROLLO, PILOTAJE Y VALIDACIÓN DE LA PRUEBA PRAP (PRUEBA DE RECONOCIMIENTO AUTOMÁTICO DE PALABRAS) EN ESTUDIANTES CHILENOS DE KINDER A TERCER AÑO BÁSICO
}

\author{
DEVELOPMENT, PILOTING AND \\ VALIDATION OF PRAP TEST \\ (AUTOMATIC WORD RECOGNITION TEST) \\ IN BASIC THIRD YEAR OF \\ KINDERGARTEN CHILEAN STUDENTS
}

Pelusa Orellana

Jill Fitzgerald Carolina Melo

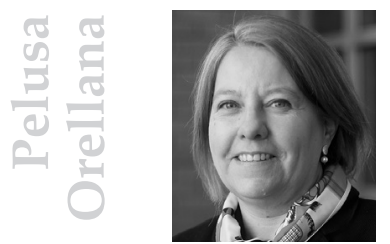

Profesora asociada e investigadora, Facultad de Educación, Universidad de los Andes, Chile. Vice Decana de Investigación de la misma facultad. Inició su carrera profesional como profesora de Inglés y de Literatura. Dicta cursos en didáctica y diagnóstico de la lectura a nivel de pregrado y post grado, y ha realizado asesorías y capacitaciones a diversas instituciones educacionales en Chile. Sus líneas de investigación se centran en el diagnóstico e intervención de dificultades lectoras, la formación de profesores en el área de la lectura, la motivación lectora y el desarrollo del ambiente letrado.

Correo electrónico: [porellan@uandes.cl]. 


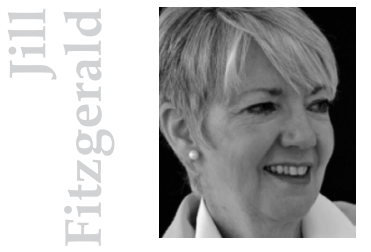

Profesora emérita e investigadora en la Universidad de North Carolina at Chapel Hill, y científico destacada de MetaMetrics en Durham, North Carolina. Inició su carrera profesional como profesora de educación primaria y especialista en lectura. Luego dictó cursos tanto en pregrado como en postgrado en las áreas de enseñanza de la lectura y escritura por más de 30 años. Ha publicado más de 100 artículos en un amplio espectro de revistas científicas de primer nivel y ha sido invitada a exponer en múltiples conferencias nacionales e internacionales. Sus líneas de investigación actuales se relacionan con la alfabetización de niños multilingües y la complejidad textual. Correo electrónico: [jfitzgerald@lexile.com].

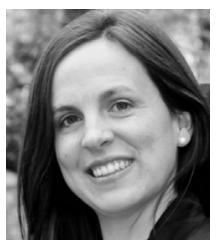

Profesora asistente e investigadora, Facultad de Educación, Universidad de los Andes, Chile. Doctoranda en la Universidad de Virgina, con el apoyo del Gobierno de Chile y la Universidad de los Andes. Máster en Psicología Educacional, Universidad de Arizona. Profesora de Educación General Básica y Licenciada en Educación, Pontificia Universidad Católica de Chile. Su área de investigación incluye evaluación de habilidades lenguaje y lectura inicial, junto con mejora de profesional docente de la etapa preescolar hasta tercer año básico. Correo electrónico: [carolina.melo.h@gmail.com]. 


\section{RESUMEN}

El presente estudio describe el desarrollo, pilotaje y validación de la prueba PRAP instrumento creado para evaluar la habilidad de reconocer palabras en español de los estudiantes de kínder a tercer año básico, en Chile. El instrumento se basa en un enfoque diagnóstico que va del todo a las partes, cuya principal premisa es que la comprensión lectora silenciosa -independientemente del nivel de dificultad textual- requiere de las habilidades de reconocimiento de palabras, comprensión verbal y procesamiento textual. En este trabajo damos cuenta de la confiabilidad, validez de constructo y el concurrente del instrumento, y constatamos que el reconocimiento automático de palabras está fuertemente relacionado a la comprensión lectora. Contar con pruebas que monitoreen el desarrollo de esta habilidad de manera confiable y válida resulta de gran utilidad para países como Chile, toda vez que este tipo de evaluaciones son escasas en el idioma español.

Palabras clave: Diagnóstico lector; reconocimiento de palabras; vocabulario visual; decodificación; automaticidad.

\section{ABSTRACT}

The current study describes the development, piloting and validation procedures for PRAP, an assessment tool created to evaluate Spanish word recognition abilities in Chilean students in grades Kindergarten through third. The central premise of the approach is that successful silent reading comprehension at every level of text difficulty requires three abilities: word identification, language comprehension, and print processing. In the current study we describe the procedures employed to establish construct and concurrent validity for the PRAP, and show that automatic word recognition is Spanish is strongly related to reading comprehension. The use of assessment tools to monitor the development of word recognition skills with validity and reliability is extremely useful in countries such as Chile, especially because there are only a handful of Spanish word recognition assessments.

Key words: Reading diagnosis; word identification; sight words; decoding; automaticity. 


\section{INTRODUCCIÓN}

Chile es uno de los países con menor índice de analfabetismo a nivel latinoamericano. Apenas un $4.2 \%$ de la población no sabe leer, lo que equivale a alrededor de 500.000 personas (Encuesta Casen, 2013). Esta cifra refleja un avance significativo en los últimos 40 años, dado que nuestro país pasó de un $11.7 \%$ de población analfabeta en los años 70, a menos de la mitad cuarenta años después. En el contexto latinoamericano, solo es superada por países como Cuba, Argentina y Uruguay, los cuales se mantienen por debajo de la línea definida por la Unesco para lo que constituye un país libre de analfabetismo.

Sin embargo, el analfabetismo funcional es el principal problema de jóvenes y adultos chilenos, cuya cifra se ha mantenido alta en los últimos quince años. Según un estudio realizado en 2013 por el Centro de Microdatos de la Universidad de Chile, un $44 \%$ de la población adulta no entiende lo que lee; $y$ en esta cifra se incluye a personas con estudios superiores. En este mismo estudio, se constató que más del $80 \%$ de los chilenos posee niveles muy incipientes de comprensión lectora, lo que significa no ser capaz de comprender textos básicos, como instrucciones simples o manuales. Esta realidad tiene graves consecuencias para el desarrollo del país en múltiples ámbitos de crecimiento y generación de oportunidades para mejorar la calidad de vida de la población (Centro de Microdatos, 2013).

Numerosos estudios confirman la fuerte correlación entre la calidad de las experiencias escolares y el nivel de desempeño en tareas de alfabetización, lo que en el caso de Chile está además fuertemente asociado al componente socioeconómico y a las experiencias escolares. Es decir, quienes poseen menos ingresos asisten a escuelas de peor calidad y, por ende, desarrollan menos sus habilidades de alfabetización. La desigualdad en el tipo de experiencias de aprendizaje de la lectura se manifiesta también en la brecha existente entre quienes tienen mejor desempeño en lectura inicial en nuestro país, y quienes logran resultados considerablemente inferiores (Mineduc, 2011).

En este sentido, urge la implementación de herramientas adecuadas de diagnóstico que posibiliten a los docentes el identificar oportunamente problemas de lectura y así reorienten su enseñanza. Ello permite otorgar mejores oportunidades de aprendizaje a los niños en todos los 
niveles socioeconómicos. Lamentablemente, a la fecha existen pocas herramientas en el idioma español que sean de fácil aplicación en el contexto escolar, ya que las pocas que existen tienen un alto costo y no siempre entregan resultados de forma oportuna.

El objetivo del presente trabajo es dar a conocer el proceso de desarrollo y validación de una prueba diagnóstica, la cual-a través de la evaluación del reconocimiento automático de palabras en españolpretende identificar posibles problemas en el desarrollo de la lectura inicial en los niveles de kinder a tercero básico. Al mismo tiempo, este artículo describe los resultados de esta prueba para una muestra croseccional de niños chilenos de establecimientos municipalizados y subvencionados entre kinder y tercero básico, entregando así una visión desde la perspectiva del desarrollo de las habilidades evaluadas.

\section{¿QUÉ SE SABE ACERCA DE LA IMPORTANCIA DEL RECONOCIMIENTO DE PALABRAS $Y$ LA LECTURA INICIAL?}

Existe considerable evidencia empírica que fundamenta la noción de que el reconocimiento de palabras posee un rol esencial en el desarrollo de la comprensión lectora. En algunos ámbitos se le denomina vocabulario visual, decodificación, o simplemente lectura de palabras. Sin embargo, el reconocimiento de palabras se refiere a «las conexiones que los lectores hacen entre el conocimiento ortográfico y fonológico de dichas palabras» (Erickson, Clendon, Cunningham, Spadorcia, Koppenhaver, Sturm \& Yoder, 2008, p. 64). Estas conexiones, que parecieran a simple vista relativamente fáciles o naturales, no lo son: requieren de un trabajo complejo de integración y síntesis que muchos niños no adquieren en la educación preescolar, si no se les proveen las oportunidades para realizarlo.

El reconocimiento de palabras se lleva a cabo de dos formas: una es la automatización, o la capacidad de leer una palabra a simple vista; es decir, en menos de un cuarto de segundo; y la decodificación, que implica unir de manera más consciente grafemas y fonemas para pronunciar la palabra y que, por ende, toma más tiempo que el reconocimiento automático. A medida que los lectores adquieren más destreza en el 
reconocimiento de palabras son capaces de leer una mayor cantidad de ellas de manera automática (Cunningham, 1993; Cunningham, Erickson, Spadorcia, Koppenhaver, Cunningham, Yoder \& McKenna, 1999). La automaticidad en la lectura de palabras facilita la comprensión, dado que permite al lector aprovechar su energía cognitiva en labores de comprensión en lugar de agotarla en el dominio del código lingüístico, mediante el análisis. En la lectura automática de palabras, el lector identifica la palabra como un todo, sin mayor análisis (Ehri, 2005). Por otra parte, la decodificación requiere de un conocimiento operativo del principio alfabético; es decir, comprender que los sonidos y grafemas y sus combinaciones operan de manera sistemática y predecible (Morrow \& Morgan, 2006). Cuando este conocimiento no se consolida en los primeros años de escolaridad, la comprensión lectora se dificulta, pues los lectores deben abordar tareas y textos cada vez más complejos (Zimmerman, Padak \& Rasinski, 2008).

La decodificación incluye una gama de estrategias, tales como separar las palabras sonido a sonido, o bien dividiéndolas en partes, para luego unirlas. Cuando los lectores adquieren mayor destreza lectora - por lo general después del segundo año de educación básica- utilizan sus nociones acerca de la estructura de las palabras para leerlas; por ejemplo, pueden identificar raíces, prefijos, sufijos o sílabas y, basándose en estos conocimientos, decodificar la palabra completa.

\section{ETAPAS EN EL PROCESO \\ DE RECONOCIMIENTO DE PALABRAS}

La adquisición de las habilidades de reconocimiento de palabras no ocurre en un momento único. Según Ehri (1995), es posible identificar cuatro etapas en el desarrollo del reconocimiento de palabras, y estos procesos ocurren mediante un análisis cuidadoso de la estructura ortográfica de cada palabra y su consiguiente representación mental, la cual puede ser más o menos automática, dependiendo de la etapa de desarrollo en la que se encuentre el lector. Así, por tanto, existe una fase pre-alfabética en la que los lectores relacionan algunos atributos visuales de las palabras, ya sea su pronunciación o su significado. Un ejemplo de ello es la relativa facilidad con la que lectores principiantes son capaces de identificar palabras tales como «ojo», que 
visualmente se asemeja en su forma: las dos «o» representan dos ojos y la «j» la nariz en el rostro de una persona. Sin embargo, estas conexiones no se basan en un conocimiento explícito de las relaciones entre fonemas y grafemas. En una segunda etapa llamada alfabético-parcial, el lector comienza a establecer conexiones entre ciertas letras y algún sonido inicial o final. Con este conocimiento básico, identifican un vocabulario visual limitado, dado que su conocimiento del nombre y/o sonido de las letras es aún incompleto. Posteriormente - y cuando ya son capaces de comprender la relación entre fonemas y grafemas en la ortografía convencional-, los lectores principiantes pueden reconocer un número importante de palabras cuyo patrón ortográfico es relativamente similar. Esta etapa se denomina alfabético-total. Aquí, el repertorio de vocabulario visual, sin embargo, se limitará principalmente a aquellas palabras que encuentran con mayor frecuencia. Finalmente, al llegar a la etapa que Ehri denomina alfabético-convencional, el lector puede identificar patrones ortográficos en múltiples palabras y generalizar su conocimiento del sistema ortográfico, con lo que se reduce de manera considerable el desgaste en el procesamiento de la información. Por lo tanto, el llegar a esta etapa final en el reconocimiento automático de palabras se vincula directamente con la adquisición de fluidez en la lectura (LaBerge \& Samuels, 1974; Moors \& De Houwer, 2006; Roberts, Christo \& Schefelbine, 2011) y, por consiguiente, con la comprensión. La automaticidad como práctica cognitiva requiere de la ejercitación, que puede acompañarse de cambios en los focos de atención, conciencia, control, velocidad y precisión (Moors et al., 2006; Nation \& Snowling, 1998).

Es importante mencionar que, a diferencia de las primeras tres etapas, que contribuyen a la capacidad del lector para identificar palabras poco frecuentes o desconocidas, la etapa de consolidación alfabética ayuda a leer las palabras que conocemos mejor, pues en esta fase se emplea la memoria o la vista para identificarlas sin mayor esfuerzo (Ehri, 2005). Otra característica importante del reconocimiento automático de palabras es que cada una de ellas se lee como una unidad, sin que haya pausas entre sus partes, y sin procesamiento secuencial de las letras.

También es importante recordar que las etapas descritas por Ehri son las mismas tanto en idiomas con ortografías transparentes, como aquéllas más opacas, aunque pueden variar en la forma 
en que se desarrollan. En el caso del español, cuya correspondencia grafema-fonema es casi perfecta, la etapa alfabético-parcial puede ser más breve, ya que la decodificación surge de manera más temprana (Cardoso-Martins, 2001).

Existe suficiente evidencia empírica para fundamentar la relación entre el reconocimiento de palabras y la comprensión (Nation \& Snowling, 1998; Perfetti, 2010; Perfetti, 1985, Adams, 1990; 1994). Por lo general, los buenos lectores poseen un repertorio amplio de palabras que pueden leer en forma automática, lo cual agiliza su lectura y les permite focalizarse en la elaboración de significado (Ehri, 2005). Por otra parte, el no alcanzar un buen nivel de desarrollo de automaticidad puede suscitar dificultades lectoras posteriores (Roberts et al., 2011; Cunningham, Schmidt, Nathan \& Raher, 2011). No obstante, el ser capaz de decodificar o reconocer palabras con relativa automaticidad no garantiza la habilidad de comprensión (Cutting \& Scarborough, 2006). De hecho se estima que, entre un 10 a un $20 \%$ de los lectores problemáticos, no pueden elaborar el significado de un texto, pese a ser capaces de decodificarlo (Leach, Scarborough \& Rescorla, 2003). Esta realidad explica el analfabetismo funcional que describimos al comienzo del presente estudio, y que da cuenta de la importancia de monitorear los procesos de comprensión, decodificación y automaticidad de forma paralela.

\section{FACTORES ASOCIADOS}

\section{A LA LECTURA DE PALABRAS}

El reconocimiento de palabras de forma más o menos fluida es una condición fundamental para la comprensión; el logro de este objetivo depende de la relación entre procesos fonológicos, memoria (corto y largo plazo) y la velocidad para acceder a representaciones fonológicas que discriminen entre una palabra y otra, o entre una palabra y una pseudopalabra. Existe suficiente evidencia, tanto empírica como teórica, acerca de los factores que inciden en la capacidad que tienen los lectores principiantes para leer las palabras que se les presentan con mayor o menor grado de éxito. Diversos modelos (e.g., doble ruta, modelo simple, recodificación fonológica, entre otros), han intentado explicar la manera en que los lectores acceden y utilizan los distintos 
mecanismos de acceso a la información, mismos que les permiten leer una palabra. En cada uno de estos modelos, los factores que detallaremos a continuación inciden de manera más o menos importante en el éxito en la lectura de las mismas.

Un primer aspecto vinculado a la lectura de palabras es la relación entre la lectura de palabras y el grado de transparencia o profundidad del idioma en que se lee. Un idioma se considera transparente cuando existe una mayor correspondencia entre sus fonemas y sus grafemas. El español es un idioma ortográficamente transparente, y el procesamiento fonológico es relativamente más sencillo que en el caso de idiomas de ortografía profunda como el inglés, donde no existe una perfecta correspondencia fonema-grafema (Cuetos \& Domínguez, 2002, Treiman \& Kessler, 2003). En el caso de las ortografías transparentes, los lectores identifican más rápidamente las palabras deduciendo el sonido de una letra a partir de su nombre (González, López, Vilar \& Rodríguez, 2013). Enfrentados a la lectura de palabras en idiomas como el español, los lectores acuden principalmente al mecanismo de recodificación fonológica para captar la palabra, sin depender tanto del procesamiento léxico u ortográfico. Lo anterior ha demostrado el alto nivel de predictibilidad que las tareas fonológicas tienen sobre el desarrollo de la lectura (National Reading Panel, 2000; Defior \& Serrano, 2011; Caravolas, Violin \& Hulme, 2005).

Otra variable analizada dice relación con la cantidad de oportunidades en que un lector se enfrenta a la lectura de una determinada palabra. Así por tanto, aquellas palabras que aparecen con más frecuencia son identificadas con mayor rapidez y facilidad, lo que eventualmente lleva a la automaticidad, permitiendo liberar energía cognitiva que se aprovecha para la comprensión (Defior, 2014; Hiebert \& Fisher, 2007). La frecuencia de exposición es un factor que incide en la lectura de palabras en todos los idiomas, independientemente del nivel de transparencia u opacidad que posean.

Un tercer aspecto a considerar dice relación con la composición de las palabras. La presencia de morfemas dentro de una palabra incide en el desempeño de la lectura temprana. En el caso de ortografías menos transparentes parece resultar más fácil leer palabras derivadas que no derivadas, dado que ante la dificultad de utilizar la correspondencia 
fonema-grafema, el lector recurre típicamente a una unidad mayor, como es el morfema, pudiendo así discriminar más eficazmente entre una palabra y otra (Jaichenco \& Wilson, 2013). La regularidad o irregularidad de determinados patrones silábicos, en conjunto con la frecuencia $u$ oportunidad para leer reiterativamente una palabra, tienen, en este sentido, un rol esencial en la adquisición de palabras nuevas. Es esperable, por ejemplo, que palabras de uso poco frecuente y de composición más bien compleja resulten más difíciles de leer para los lectores iniciales, y probablemente resulta complejo aislar un fenómeno del otro. También en el campo de los aspectos estructurales de una palabra se incluye su longitud, composición silábica, y en el caso del español, la acentuación (Defior, Fonseca, Gottheil, Aldrey, Pujals, Rosa, Jiménez \& Serrano, 2007).

Variables menos analizadas, y que son de considerable importancia para una lectura cada vez más automática de palabras son: la edad de adquisición, la extensión y el grado de familiaridad. Alija y Cuetos (2007) demostraron que la edad en que un niño conoce una palabra incide, de manera significativa, en el reconocimiento visual de palabras en el idioma español. A su vez, podría esperarse que las interacciones a través del lenguaje oral, al que están expuestos los niños antes de ingresar al sistema escolar, incidan precisamente en la edad a la cual adquieren una palabra y cuán familiarizados están con ella, incluso desde un punto de vista semántico.

\section{DESCRIPCIÓN DEL INSTRUMENTO: LA PRUEBA PRAP}

La prueba PRAP (Prueba de Reconocimiento Automático de Palabras) fue diseñada como un componente de una plataforma para el diagnóstico de dificultades de lectura temprana. Su objetivo es evaluar la capacidad de reconocer en una pantalla, visualmente, una serie de palabras que se presentan al estudiante, y que este debe ser capaz de leer, ya sea en forma automática o mediante la decodificación. Si el estudiante no logra leer la palabra en forma automática; es decir, en menos de un cuarto de segundo, la palabra vuelve a aparecer y el estudiante tiene hasta cinco segundos para leerla, usando la decodificación. 


\section{Elaboración de la Prueba PRAP}

Para crear el instrumento se utilizó una base de datos de tres mil palabras seleccionadas por el Ministerio de Educación [de Chile], a partir de los textos escolares y lecturas asignadas a los cursos del primer ciclo básico (primer a cuarto año). La lista de palabras se ordenó por grado de dificultad creciente en función de su complejidad silábica (Villalón, 2008). Bajo este constructo se conformaron 13 criterios según los cuales fue posible agrupar palabras según su complejidad. Por ejemplo: las combinaciones más sencillas son los patrones CVC o CVCV (ejemplo: pan, taco) que resultan más fáciles de decodificar para los lectores principiantes, mientras que en el otro extremo se ubicaron palabras con tres sonidos consonantes (ejemplo: sombrero), con los diptongos «que-qui» o «güe-güi» (ejemplo: esquina), o la combinación «ce-ci» (ejemplo: encima) que resultan más difíciles de leer a primera vista. Con base a estos criterios (ver Anexo 1) se generaron 15 listas con 20 palabras cada una. De este modo, el instrumento incluye 300 palabras provenientes de la base generada por el Ministerio de Educación. Cada lista corresponde más o menos a uno de los criterios de complejidad antes descritos.

Con esta primera selección de palabras se realizó un pilotaje con 50 niños de los niveles escolares antes mencionados, lo que permitió explorar de manera preliminar si los niños de los distintos cursos eran capaces de leer las palabras y asegurar que estas hubieran estado ordenadas, efectivamente, en función de su nivel de complejidad. Se contrastó el orden original de las palabras en relación con el nivel de dificultad, con la dificultad que presentaron en el pilotaje para los niños, y se hicieron pequeños ajustes para lograr un listado que realmente reflejara dicha complejidad, tanto desde una perspectiva teórica como empírica. Se ordenaron las palabras de acuerdo con el promedio de respuestas correctas de cada una en la tarea de reconocimiento automático y luego se comparó este listado con el original. Los ajustes consistieron principalmente en re ordenar aquellas palabras que, de acuerdo con el resultado de pilotaje, estaban en un listado de nivel más alto o más bajo de complejidad que la dificultad que realmente presentaron para los niños.

\section{Características y administración del instrumento}

En la prueba PRAP, las palabras aparecen una a una en la pantalla de una tablet o computadora. El alumno debe leerla en forma 
automática, y si no es capaz de hacerlo, el evaluador vuelve a presentarle la palabra en la pantalla presionando el botón «volver». Mientras el alumno lee las palabras, el evaluador va registrando en una esquina de la pantalla la cantidad de palabras leídas correctamente en menos de un cuarto de segundo, y aquellas que son decodificadas. Las palabras están agrupadas en 15 listas de 20 palabras cada una, y aparecen en un orden de complejidad creciente, por lo tanto es esperable que los niños tengan menor dificultad para leer las primeras palabras que las presentadas hacia el final de la prueba. Sin embargo, si un alumno no es capaz de leer correctamente 4 o más palabras de una lista, la prueba finaliza automáticamente. Cada palabra leída en forma automática se califica con un punto, mientras que cada palabra leída mediante análisis o decodificación recibe 0.5 puntos. Así, la prueba posee un puntaje máximo de 300 puntos.

\section{METODOLOGÍA}

\section{Participantes}

Ciento treinta y nueve estudiantes chilenos provenientes de tres establecimientos educacionales municipales y particulares pagados, de la Región Metropolitana, participaron en el pilotaje del instrumento. Se trata, por tanto, de una muestra por conveniencia que incluyó a estudiantes chilenos cursando kínder, primero, segundo y tercer año básico. De los 139 participantes iniciales, solo fue viable obtener resultados de ambas mediciones (inicio y final de año) para 79. Tampoco fue posible acceder a la información demográfica relacionada con ingreso familiar para así determinar el nivel socioeconómico de los participantes; sin embargo, se consideró el tipo de dependencia del establecimiento como un acercamiento al nivel socioeconómico. En este sentido, la muestra está constituida por 51 estudiantes de colegio particular pagado y nivel socioeconómico alto, mientras que 88 corresponden a establecimientos municipalizados y pertenecen a familias de nivel socioeconómico medio bajo. Del total de participantes, 79 eran niños y 60 niñas, y se distribuyeron por curso de la siguiente forma: 45 niños y niñas en kínder, 31 en primero, 21 en segundo y 42 en tercero. 


\section{Aplicación}

El instrumento fue aplicado en dos oportunidades: una vez al comienzo del año escolar (fines de marzo) y, posteriormente, una segunda vez hacia fines de noviembre. Las dos aplicaciones estuvieron a cargo de tres evaluadoras debidamente entrenadas, además de dos de las investigadoras. El entrenamiento consistió en una sesión de dos horas de presentación del instrumento y práctica, marcando las respuestas correctas e incorrectas en la pantalla. Dado que las instrucciones para la prueba estaban grabadas en el dispositivo y los niños las escuchaban directamente desde el ipad, el rol del evaluador era solo marcar las respuestas correctas e incorrectas, por lo que no se estimó que se requiriera mayor nivel de entrenamiento.

Para la aplicación de la prueba, cada alumno se sentó frente a la pantalla de una tablet con la evaluadora a su lado. Las instrucciones de la prueba se presentaron a través de audio y la evaluadora constató que el estudiante comprendiera lo que debía hacer. Las palabras se mostraron a los estudiantes en la pantalla de la tablet, siguiendo los procedimientos del modelo de Cunningham et al. (2011), que consiste en proyectar cada palabra durante un cuarto de segundo, tiempo durante el cual el niño debe leerla. Si lee la palabra en ese lapso de tiempo, aparece una nueva palabra, y así sucesivamente. Si no es capaz de leerla en forma automática, el evaluador hace click en el ícono «volver» y la palabra vuelve a aparecer por 4 a 5 segundos, lo que da tiempo suficiente para que el niño utilice estrategias de análisis y decodificación para leer la palabra. Si en esta segunda oportunidad el niño no logra leerla, el evaluador presiona el ícono «volver» y aparece la palabra siguiente.

\section{Asignación de puntaje}

A cada palabra leída en un cuarto de segundo se le asigna un punto, mientras que por cada palabra leída en un tiempo mayor, el niño recibe medio punto. Esto permite identificar cuáles son las palabras que forman parte del vocabulario visual del niño y cuáles son más complejas de leer. Así, por ejemplo, el máximo de puntaje por leer la totalidad de palabras en una lista es de 20 puntos (uno por cada palabra leída en forma automática). El niño avanza de una lista más sencilla a otra más 
compleja siempre y cuando obtenga al menos 17 puntos en la lista anterior. Si su puntaje es inferior a 17, el evaluador termina la aplicación en dicha lista y palabra.

\section{RESULTADOS Y DISCUSIÓN}

En esta sección presentamos y analizamos los principales resultados del proceso de pilotaje y validación de la prueba PRAP. En primer lugar detallamos el análisis de confiabilidad, y en un segundo apartado nos referiremos a los análisis para establecer la validez de constructo del instrumento aplicado.

\section{Confiabilidad}

La confiabilidad es el grado en el que un determinado instrumento de evaluación mide consistentemente aquello que desea medir. En el caso de la prueba PRAP, se pretende establecer que esta prueba es un instrumento confiable para medir la capacidad de reconocer palabras en español de manera automática (a primera vista), o bien mediante la decodificación o análisis, desde kínder a tercer año básico. Para determinar dicha confiabilidad se realizaron análisis de correlación entre los ítems de la prueba, en una muestra de 142 estudiantes de los cursos kínder, primero, segundo y tercero básico evaluados al inicio del año escolar 2012. Las correlaciones arrojaron un alfa de Cronbach de .97, ratificando la consistencia interna del instrumento.

En segundo lugar, se realizaron correlaciones test-retest entre los puntajes de los niños al inicio y al finalizar el año escolar, las cuales fueron significativas para el reconocimiento automático de palabras, obteniendo una correlación de .7 ( $\mathrm{p}<.01)$. Esto permite establecer que el instrumento, en cuanto a reconocimiento automático de palabras, muestra una estabilidad de los puntajes en el tiempo. No obstante, este no fue el caso para la correlación del test re-test de decodificación o lectura de palabras mediante análisis. La falta de una correlación significativa poderosa entre comienzo y fin de año para la decodifciación es esperable, ya que en la medida que la lectura se torna más automática, habrían menos palabras que el lector necesitaría decodificar (disminuyendo, así, el número de ítems para el 
test de decodificación); al mismo tiempo, el lector puede enfrentarse a palabras de mayor complejidad que anteriormente y presentar un mayor número de respuestas incorrectas. Si bien para reconocimiento automático de palabras se esperaría que un niño fuese capaz de leer más palabras en forma automática hacia fines del año escolar que a comienzos del mismo, el caso de la decodificación es distinto. La evolución de los puntajes no es necesariamente estable, pues dependerá de si a lo largo del año ha estado expuesto a textos de creciente complejidad, y por tanto ha debido utilizar estas estrategias para leerlas. Si aumenta el número de palabras leídas por la vía de la decodificación, significa que el lector está utilizando esta estrategia de manera exitosa, pero al mismo tiempo está siendo expuesto a textos con palabras que le son menos familiares. En síntesis: automaticidad y decodificación son procesos fuertemente relacionados e importantes para el desarrollo del reconocimiento de palabras (Erickson et al., 2008). Mientras más eficiente es la decodificación de palabras desconocidas, mayor es la capacidad de leer palabras en forma automática (Ehri, 1992), y de manera inversa, a medida que se adquiere mayor automaticidad en la lectura, aumenta también la eficiencia en la decodificación de palabras menos conocidas (Cunningham, 2000).

\section{Validez de constructo}

En el presente estudio damos cuenta de la validez de constructo de la prueba PRAP. La validez de constructo incluye dos categorías o subtipos: validez convergente (la relación entre medidas teóricamente similares) y la validez discriminante (supone que mediciones de constructos que en teoría no se relacionan, tampoco lo hacen en la práctica).

Para analizar la validez convergente consideremos las correlaciones entre los distintos ítems explicada anteriormente, la cual arroja un alfa de Cronbach de .97; es decir, muestra una alta convergencia de los ítems en torno al constructo de identificación de palabras. Por otra parte, para establecer la validez discriminante entre la medida de reconocimiento automático y decodificación, es posible observar que, de acuerdo con lo esperado, la correlación entre ambas medidas, tanto en la primera como en la segunda aplicación, son bastante bajas (.152 y .185 , respectivamente). 
Otra manera de examinar la validez de constructo consiste en comparar las medias y desviaciones estándar de cada nivel evaluado, y examinar si existe una progresión de estas habilidades de acuerdo con el nivel escolar de los niños. Las Tablas 1 y 2 muestran los resultados desagregados por nivel en cada período evaluado, tanto para reconocimiento automático de palabras como para decodificación.

En estos resultados es posible observar que a medida que aumenta el curso (o grado escolar) crece también el promedio de palabras leídas en forma automática respecto del curso anterior, en el caso del pre-test (marzo). Este resultado es esperable, pues cuando un niño está aprendiendo a leer, a medida que avanza en el sistema escolar la frecuencia de exposición a palabras de creciente complejidad es mayor, por lo tanto aumenta su repertorio de palabras reconocidas en forma automática, y se enfrenta a palabras nuevas para las cuales utiliza la decodificación o el análisis como herramientas de lectura. La Tabla número 1 presenta las medias, las desviaciones estándar y los puntajes brutos mínimos y máximos para el reconocimiento automático de palabras, evaluado al inicio y fin del año escolar. En kínder y primer año básico, existe un incremento en las palabras leídas tanto de forma automática como mediante la decodificación. Este incremento en el número de palabras leídas ocurre también si se comparan los resultados en los dos períodos evaluados. En segundo y tercer año básico, observamos un aumento en el número promedio de palabras leídas en forma automática respecto de los cursos anteriores por lo que, para los cuatro cursos evaluados, es posible establecer que el puntaje de palabras leídas aumenta en función del curso.

Sin embargo, para segundo y tercer año básico, se incrementa solo el número de palabras decodificadas y se observa una disminución importante de palabras leídas en forma automática, en particular en segundo año básico. No obstante, podría esperarse que los incrementos más significativos se dieran en los primeros cursos (primero y segundo básico), pues son los niveles en que la mayoría de los niños adquieren el proceso lector más plenamente. Por otra parte, el descenso en la lectura automática de palabras en tercero 
básico se contrasta con el incremento de palabras decodificadas, que pasan de 91.63 a 108.21, lo cual podría explicarse porque, tras alcanzar un adecuado nivel de automaticidad, y habiendo sido expuestos a textos con mayor repertorio de palabras nuevas, los lectores recurren a la decodificación para leer las palabras desconocidas (Cunningham, 1995).

\begin{tabular}{|c|c|c|c|c|}
\hline \multicolumn{5}{|c|}{ RECONOCIMIENTO AUTOMÁTICO (N=79) } \\
\hline & Mínimo & Máximo & $\mathrm{M}$ & SD \\
\hline \multicolumn{5}{|c|}{ Reconocimiento automático de palabras } \\
\hline \multicolumn{5}{|l|}{ Kindergarten } \\
\hline Marzo & 0 & 11 & 1.03 & 2.35 \\
\hline Noviembre & 0 & 63 & 3.54 & 12.47 \\
\hline \multicolumn{5}{|l|}{1} \\
\hline Marzo & 0 & 220 & 20.93 & 54.26 \\
\hline Noviembre & 0 & 298 & 53.5 & 79.96 \\
\hline \multicolumn{5}{|l|}{2} \\
\hline Marzo & 6 & 235 & 111.86 & 83.74 \\
\hline Noviembre & 0 & 173 & 63.73 & 56.38 \\
\hline \multicolumn{5}{|l|}{3} \\
\hline Marzo & 32 & 299 & 155.3 & 91.63 \\
\hline Noviembre & 33 & 299 & 141.92 & 108.21 \\
\hline
\end{tabular}




\begin{tabular}{|c|c|c|c|c|}
\hline \multicolumn{5}{|c|}{$\begin{array}{l}\text { TABLA 2. ESTADÍSTICAS DESCRIPTIVAS PRAP } \\
\text { DECODIFICACIÓN O ANÁLISIS ( } \mathrm{N}=79 \text { ) }\end{array}$} \\
\hline Kindergarten & $(N=26)$ & & & \\
\hline Marzo & 0 & 3 & .30 & .77 \\
\hline Noviembre & 0 & 8.5 & 3.46 & 3.20 \\
\hline 1 & $(\mathrm{~N}=16)$ & & & \\
\hline Marzo & 0 & 6.5 & 2.00 & 1.89 \\
\hline Noviembre & 0 & 9.5 & 5.34 & 3.11 \\
\hline 2 & $(N=11)$ & & & \\
\hline Marzo & 0 & 10 & 3.09 & 3.23 \\
\hline Noviembre & 0 & 26 & 7.18 & 6.91 \\
\hline 3 & $(N=26)$ & & & \\
\hline Marzo & 0 & 6 & 2.26 & 1.83 \\
\hline Noviembre & 0 & 17.5 & 5.13 & 4.33 \\
\hline
\end{tabular}

Como se aprecia a partir de la Tabla 1, la distribución de los puntajes muestra un gran rango, especialmente en el caso del reconocimiento automático de palabras entre los alumnos de primer y tercer año básico, donde el rango varía entre 0 y 298, y 33 y 299, puntos respectivamente. Esto quiere decir que en ambos niveles hay niños que no pueden leer ninguna palabra en forma automática y otros que poseen un vocabulario visual de casi 300 palabras. En el caso de los resultados para primer año básico, la media se ubica en torno a los 50 puntos; es decir, logran $1 / 6$ del puntaje total, mientras que para tercer año básico se alcanza una media equivalente a reconocer el $50 \%$ de las palabras.

Por último, realizamos un proceso de validación concurrente para la prueba PRAP al examinar su correlación con una prueba de comprensión de lectura silenciosa aplicada a 119 niños de la muestra. El objetivo de este análisis fue establecer si los resultados en la prueba 
de reconocimiento automático de palabras y de decodificación PRAP, están relacionados al desempeño en comprensión de lectura. Múltiples estudios en el mundo anglosajón han demostrado que existe esta relación en el idioma inglés; más aún: estas investigaciones ratifican que el reconocimiento automático de palabras es un importante predictor de la comprensión lectora posterior (Fuchs, Fuchs, Hosp \& Jenkins, 2001; Hasbrouck \& Tindal, 2006). En este estudio, la comprensión de lectura silenciosa se midió a través de la utilización de la prueba Dialect 1 , que, al igual que PRAP, forma parte de la batería Dialect para el diagnóstico de dificultades lectoras (Orellana, Melo, Fitzgerald \& Cunningham, 2013; Orellana \& Melo, 2015). Esta prueba en particular emplea el marco Lexile para la medición de comprensión lectora. El sistema Lexile - desarrollado por Metametrics en North Carolina, Estados Unidoses una unidad de medida que reconoce el nivel lector del estudiante a través de su comprensión de lectura silenciosa, e identifica el nivel del texto correspondiente a las habilidades del lector. Esta medida ha sido validada en Estados Unidos (en inglés y español) y es ampliamente utilizada para identificar el nivel de comprensión de lectura y el nivel adecuado de dificultad textual de millones de textos en ambos idiomas (Sanford, 2000). La prueba se aplicó a 119 estudiantes evaluados a inicios del año escolar 2014 y mostró una correlación significativa con PRAP de un alfa de Cronbach de $.745^{* *}$, lo que permite concluir que ambos instrumentos miden constructos que están fuertemente relacionados. Una mejora en este caso en el reconocimiento y lectura de palabras está altamente relacionada a un progreso en comprensión lectora. Este hallazgo es muy relevante para el contexto hispanoparlante ya que confirma una relación que muchos investigadores han constatado para el idioma inglés pero que, hasta donde nosotros sabemos, no ha sido mayormente estudiada en el idioma español.

\section{Resultados descriptivos de la prueba PRAP}

Al observar el porcentaje de alumnos que responden correctamente cada ítem, notamos que para las 20 palabras del primer tramo, el porcentaje de palabras leídas en forma automática por los alumnos varía de 48 a $72.5 \%$, siendo la palabra «mamá» la que es leída en forma correcta por un mayor porcentaje de alumnos en menos de un cuarto de segundo. La palabra «nada» es la que obtiene el porcentaje más bajo 
de lecturas correctas en este conjunto de palabras. Otra observación interesante es que el porcentaje de alumnos capaz de leer cada palabra en forma correcta va disminuyendo de una lista a otra, lo que se presumía si las palabras estaban adecuadamente ordenadas según grado de dificultad. Así, por ejemplo, en el segundo tramo, el rango de porcentajes de lecturas correctas va de $47.3 \%$ (palabra «calle») a $41.2 \%$ (palabra «roca»). En el otro extremo de palabras se encuentran las 20 palabras de la lista 15, consideradas como más complejas desde un punto de vista silábico, y en las que el rango de porcentaje de lecturas correctas va del 5.4 al $4.7 \%$. Dentro de las palabras que presentaron mayor dificultad de lectura para los estudiantes están las combinaciones de vocales con letra «c» como por ejemplo: «aceptar», «anciano», «importancia», «noticia». También en el rango de 5.4\% de lectura correcta están las combinaciones «gui», «que», «quie». Estos resultados hacen pensar que el orden en que las palabras fueron presentadas refleja, efectivamente, su creciente nivel de complejidad para lectores de este rango etario.

En el caso de las palabras que los alumnos leen por vía de la decodificación o análisis, la dispersión de resultados es inferior a la de las leídas en forma automática, y las medias para todos los cursos (salvo kindergarten), van de los 2 a 7 puntos; es decir, del total de palabras leídas, en promedio, un máximo de 14 fueron leídas empleando estos métodos. A la luz del planteamiento de Ehri (2005), se podría pensar que entre los estudiantes evaluados de este nivel predomina el uso de la memoria para identificar palabras. El bajo promedio de logro y el rango de valores mínimos y máximos en esta habilidad dan cuenta de que aún existen diferencias importantes en el grado de desarrollo de la automaticidad entre los alumnos de un mismo curso o nivel lo que, al correlacionarse con el desempeño en comprensión lectora silenciosa, ayuda a entender por qué muchos niños todavía no logran comprender lo que leen.

Al analizar las palabras correctamente decodificadas por los evaluados, las palabras que resultan más difíciles de leer son aquellas con combinaciones de doble consonante (por ejemplo: empleado, escuela, industria, sangre), y palabras con combinaciones $\mathrm{CVCV}$, son las decodificadas por un mayor porcentaje de niños. A modo de ejemplo, la palabra «toma» es leída por un $40.3 \%$ de los niños evaluados, mientras 
que las palabras con doble consonante como «empleado», son leídas en promedio por un $7.1 \%$ de los niños. Las combinaciones CV y CVC son también las que obtienen mayores porcentajes de lectura por esta vía. Estos resultados muestran que, al igual que para la lectura automática, el orden de las palabras también está bien calibrado en función de la decodificación, y por lo tanto, las palabras que resultan complicadas de leer en forma automática, también son difíciles de decodificar para los mismos alumnos.

Con el fin de examinar cómo se desarrollan las habilidades evaluadas a lo largo de la etapa escolar inicial (kínder a tercero básico) se examinaron las diferencias en los puntajes obtenidos en los distintos niveles en los períodos de inicio y final del año. En primer lugar se realizaron análisis de varianza para reconocimiento automático, los que resultaron ser significativos tanto en marzo, $\mathrm{F}(3,100)=31.05, \mathrm{p}=.000$, como noviembre, $\mathrm{F}(3,82)=10.898, \mathrm{p}=.000$. En el caso de la decodificación, los resultados fueron significativos en marzo, $\mathrm{F}(3,100)=12.335$, $\mathrm{p}=.000$, pero no noviembre, $\mathrm{F}(3,82)=2.170, \mathrm{p}=.09$. Los resultados del análisis de varianza de los totales de la muestra mostraron diferencias significativas para el reconocimiento automático en los distintos niveles: $\mathrm{F}(3,110)=29.89, \mathrm{p}=.000$. El test Tukey post hoc mostró que los niños de kínder tuvieron un desempeño significativamente inferior a los primeros básicos $(\mathrm{M}=8.32, \mathrm{CI} 2.29,14.349)$, segundos básicos $(\mathrm{M}=70.667, \mathrm{CI} 46.78,94.55)$ y terceros básicos $(\mathrm{M}=160.53$, CI 128.58, 192.48); mientras que los primeros básicos $(\mathrm{M}=54.57, \mathrm{CI}=30.61,78.53)$ y segundos básicos obtuvieron puntajes significativamente inferiores a los alumnos de tercer año básico. No se encontraron diferencias significativas entre primeros y segundos básicos, ni entre alumnos de kínder y primer año básico.

\section{CONCLUSIONES}

Numerosos estudios confirman que la automaticidad en el reconocimiento de palabras es una habilidad fundamental para un adecuado desarrollo de la comprensión lectora (Fuchs, Fuchs, Hosp \& Jenkins, 2001; Hasbrouck \& Tindal, 2006) y un consistente predictor de las dificultades lectoras (Good, Simmons \& Kame'enui, 2001; Fletcher, Lyon, Fuchs \& Barnes, 2007; Vellutino, Fletcher, Snowling \& Scanlon, 2004). 
Durante el proceso de adquisición de esta habilidad, los niños desarrollan una serie de mecanismos básicos de aprendizaje que relacionan aspectos perceptivo-visuales, como la asociación grafema-fonema, memorísticos y ortográficos. Sin embargo, existen también factores individuales que explican las diferencias en el grado de automaticidad en la lectura que cada niño adquiere. Estas diferencias tienen que ver con el modo en que aspectos cognitivos, fonológicos, maduracionales y de memoria verbal interactúan a la hora de enfrentarse a un texto. El poder diagnosticar a tiempo cualquier dificultad en el procesamiento de información que facilite o dificulte la automaticidad es de gran utilidad para implementar estrategias de intervención tendientes a aumentar el número de palabras que un niño es capaz de leer a golpe de vista.

La Prueba de Reconocimiento Automático de Palabras (PRAP) es un instrumento válido y confiable que permite al profesor conocer el repertorio de palabras de uso frecuente que un niño es capaz de leer en forma automática y aquellas que lee mediante el análisis o decodificación. Este conocimiento es muy valioso a la hora de planificar actividades que intenten la ejercitación sistemática de la automaticidad, o bien que entreguen al niño un modelaje directo y explícito de las estrategias que contribuyen a aumentar la velocidad de reconocimiento visual. La decodificación es una tarea ardua que muchas veces cansa y frustra a los primeros lectores, pues deben destinar gran parte de su energía cognitiva al procesamiento del código, con lo que la elaboración de significado a través de la comprensión queda por lo general para un segundo plano. Al facilitar la lectura fluida mediante la automaticidad, la acuciosidad y la prosodia, el mayor porcentaje de capacidad cognitiva se reserva para la comprensión que es, en definitiva, el principal objetivo de la lectura.

A través de este estudio, hemos dado a conocer el proceso de desarrollo y validación de un instrumento de diagnóstico de dificultades lectoras basado en el reconocimiento automático de palabras y la decodificación. Al mismo tiempo, hemos mostrado datos descriptivos y comparativos en cuanto al desarrollo de estas habilidades lectoras entre los grados de kínder a tercero, lo que constituye un aporte para la comprensión del desarrollo de la lectura en la etapa escolar en el idioma español. Asimismo, constatamos con esta muestra que el reconocimiento automático de palabras está fuertemente relacionado a la 
habilidad de comprensión lectora, consistentemente con la teoría. Este último hallazgo presenta un aporte relevante en el contexto hispanoparlante, dado la escasez de estudios que examinen los factores involucrados en la comprensión lectora en el idioma español. De acuerdo con nuestros resultados podemos concluir que el reconocimiento de palabras es posible de medir de manera válida y confiable, y que está fuertemente relacionado a la comprensión lectora.

\section{ANEXO 1 \\ CRITERIOS DE SELECCIÓN DE PALABRAS}

\section{CVC o CVCV}

Palabras con letras sin doble sonido (ch, ll-rr) ni confusiones visuales (d-b-q-t), o auditivo fonémica (ch-j-g). Monosílabos.

\section{VC o CV-CV}

Lletras con confusión visual o auditivo fonémicas, palabras que tengan: $r, \tilde{n}, c$, h con sonido / $\mathrm{k}$ /

3. Sílaba compleja CVC, VC

Dos sílabas.

Palabras con confusión auditivo fonémica: J, 1l, ch, y, x.

Con confusión visual: $\mathrm{d}, \mathrm{b}$.

4. Palabras tres sílabas, CVC, VC

4a. Palabra de sílaba directa, indirecta o compleja con 4 sílabas o más.

4b. Palabras mono o bisílabas con diptongo.

5. Polisílabas con diptongo.

6. Palabras mono o bi o tri sílabas, con una sílaba con doble consonante.

7. Palabras de 4 o más sílabas con doble consonante. 
8. Palabras de bi o tri sílabas con dos doble consonante.

9. Palabras de más de 4 sílabas con dos doble consonante.

10. Palabras con diptongo, una y dos doble consonante.

11. Palaabras con triple consonante.

12. Palabras con que-qui y gue gui.

13. Palabras con ce ci.

\section{TABLA COMPLEJIDAD SILÁBICA}

- Reconocimiento de letras $>a$

- Sílaba directa CV > la

- Sílaba indirecta $\mathrm{VC}>\mathrm{el}$

- Sílaba compleja CVC > los

- Diptongo CVV > mue

- Sílaba compleja diptongada CVVC > mien

- Grupo consonántico CCV > bla

- Grupo consonántico diptongado CCVVC > cruen

- Que-qui, ce-ci

Diptongo: $\mathrm{u}$, o, e son vocales fuertes, se separan cuando se encuentran juntas en una sílaba diptongada. 


\section{REFERENCIAS}

Adams, M.J. (1990). Beginning to read. Thinking and learning about print. Boston, MA: MIT Press.

Adams, M.J. (1994). «Modeling the connections between word recognition and reading», en R.B. Ruddell; M.R. Ruddell, \& H. Singer (eds.). Theoretical models and processes of reading. Newark, DE: International Reading Association (4 ${ }^{\text {th }}$ ed., 838-863).

Alijam M. y Cuetos, F. (2006). «Efectos de las variables léxico-semánticas en el reconocimiento visual de palabras». Psicothema, 18 (3), 485-491.

Caravolas, M.; Volin, J. y Hulme, C. (2005). «Phoneme awareness is a key component of alphabetic literacy skills in consistent and less consistent orthographies: Evidence from Czech and English children». Journal of Experimental Child Psychology, 92, 107-139.

Cardoso-Martins, C. (2001). «The reading abilities of beginning readers of Brazilian Portuguese: Implications for a theory of reading acquisition». Scientific Studies of Reading, 5, 289-317.

Centro de Microdatos (2013). Segundo Estudio de Competencias Básicas de la Población Adulta. Santiago: Universidad de Chile.

Cuetos, F. y Domínguez, A. (2002). «Efecto de la pseudohomofonía sobre el reconocimiento de palabras en una lengua de ortografía transparente». Psicothema, 14(4), pp. 754-759.

Cunningham, J.W. (1993). «Whole-to-part reading diagnosis». Reading and Writing Quarterly, 9: 31-49. Overcoming Learning Difficulties.

Cunningham, J.W.; Erickson, K.A.; Spadorcia, S.A.; Koppenhaver, D.; Cunningham, P.M.; Yoder, D.E. \& McKenna, M.C. (1999). «Assessing decoding from an onset-rime perspective». Journal of Literacy Research, 31: 391-412. 
Cunningham, A. E.; Nathan, R. \& Schmidt, K. (2011). «Orthographic processing: Issues and relationships to phonological processing in beginning word recognition». In M.L. Kamil; P.D. Pearson; E.B. Moje, \& P. Afflerbach (eds.), Handbook of Reading Research, vol. IV (pp. 259-285). Mahwah, NJ, US: Lawrence Erlbaum Associates Publishers.

Cunningham, P. (1995). Phonics they use: Words for reading and writing, $2^{\text {nd }}$ ed. New York: Harper Collins College Publishers.

Cunningham, P. (2000). Phonics they use: Words for reading and writing, $3^{\text {rd }}$ ed. Boston, MA: Allyn \& Bacon.

Cutting, L.E. \& Scarborough, H.S. (2006). «Prediction of reading comprehension: relative contributions of word recognition, language proficiency and other cognitive skills can depend on how comprehension is measured». Scientific Studies of Reading, 10(3), pp. 277-299.

Defior, S. y Serrano, F. (2011). «Procesos fonológicos explícitos e implícitos, lectura y dislexia». Revista de Neuropsicología, Neuropsiquiatría y Neurociencias, 11(1), pp. 79-94.

Defior, S. (2014). «Procesos implicados en el reconocimiento de las palabras escritas». Aula, 20, pp. 25-44.

Ehri, L. (1992). «Reconceptualizing the development of sight word reading and its relationship to recoding». In P.B. Gough; L.C. Ehri, \& R Treiman (eds.), Reading Acquisition. Hillsdale, NJ: Erlbaum (pp. 107-143).

Ehri, L. (1995). «Phases of development in learning to read words by sight». Journal of Research in Reading. 18, 116-125.

Ehri, L. (2005). «Learning to read words: theory, findings, and issues». Scientific Studies of Reading, 9(2), 167-188. 
Erickson, K.A.; Clendon, S.A.; Cunningham, J.W.; Spadorcia, S.A.; Koppenhaver, D.A.; Sturm, J. \& Yoder, D. E. (2008). «Automatic Word Recognition: the validity of a universally accessible assessment task». Augmentative and Alternative Communication, 21(1), 64-75.

Fletcher, J.M.; Lyon, G.R.; Fuchs, L.S. \& Barnes, M.A. (2007). Learning disabilities: From identification to intervention. New York: Guilford.

Fuchs, L.S.; Fuchs, D.; Hosp, M.K. \& Jenkins, J.R. (2001). «Oral reading fluency as an indicator of reading competence: A theoretical, empirical and historical analysis». Scientific Studies of Reading, 5(3), 239-256.

González S., R.M.; López, S.; Vilar, J. y Rodríguez, A. (2013). «Estudio de los predictores de la lectura». Revista de Investigación en Educación, 11(2), pp. 98-110.

Good, R.H.; III, Simmons, D.C. y Kame'enui, E.J. (2001). «The importance and decision-making utility of a continuum of fluency-based indicators of foundational reading skills for third grade high-stakes outcomes». Scientific Studies of Reading, 5(3), 257288.

Hasbrouck, J. \& Tindal, G.A. (2006). «Oral reading fluency norms: A valuable tool for reading teachers». The Reading Teacher, 59, 636-644.

Hiebert, E. \& Fisher, C. (2007). «Critical word factor in texts for beginning readers». The Journal of Educational Research, 101, 1, pp. 1-10.

Jaichenko, V. y Wilson, M. (2013). «El rol de la morfología en el proceso de aprendizaje de la lectura en español». Interdisciplinaria, vol. 30, no. 1, pp. 85-99. 
LaBerge, D. \& Samuels, S.J. (1974). «Toward a theory of automatic information processing in reading». Cognitive Psychology, 6, 293-323.

Ministerio de Educación de Chile (2011). Resultados PISA 2009 Chile, Competencias de los Estudiantes Chilenos de 15 años en Lectura, Matemáticas y Ciencias.

Ministerio de Desarrollo Social de Chile. (2013). Encuesta de Caracterización Socioeconómica Nacional, Casen.

Moors, A. \& De Houwer, J. (2006). «Automaticity: a theoretical and conceptual analysis». Psychological Bulletin, vol. 132, no. 2, pp. 297-326.

Morrow, L. \& Morgan, L. (2006). «Phonics: explicit and meaningful instruction». In C. Cummins (ed.). Understanding and implementing reading first initiatives: The changing role of administrators (pp. 31-41). Newark, DE: International Reading Association.

Nation, K. \& Snowling, M.J. (1998). «Semantic processing and the development of word recognition skills: Evidence from children with reading comprehension difficulties». Journal of Memory and Language, 39, 85-101.

Orellana, P. \& Melo, C. (2015). «Dialect: Integrating technology and reading assessment to diagnose Spanish reading difficulties». Journal of Literacy and Technology, 16 (1), 38-66.

Orellana, P.; Melo, C. y Fitzgerald, J. (2014). «La relación entre la comprensión lectora silenciosa y sus subprocesos en niños con dificultades lectoras tempranas». Conferencia Internacional IRECE (Investigación en educación, Universidad Diego Portales). Santiago, enero, 8-10.

Outón, P. y Suárez, A. (2011). «Las dificultades de exactitud y velocidad lectoras en escolares de segundo de educación primaria». Revista de Investigación en Educación, 9(2), 153-161. 
Perfetti, C. (1985). Reading Ability. New York: Oxford University Press.

Perfetti, C. (2010). «Decoding, vocabulary, and comprehension. The Golden triangle of reading skill». In McKeown, M. G. \& Kucan, L. (eds.), Bringing reading research to life. New York: The Guilford Press.

Roberts, R.A.; Christo, C. \& Shefelbine, J.A. (2011). «Word Recognition». In M. Kamil; D. Pearson; E. Birr Moje \& P. Afflerbach (eds.), Handbook of Reading Research, volume IV (pp. 229-258). New York: Routledge.

Rayner, K.; Foorman, B. F.; Perfetti, C.A.; Pesetsky, D. \& Seidenberg, M.S. (2001). «How psychological science informs the teaching of reading». Psychological Science in the Public Interest, 2, 31-74.

Sanford, E. (2000). «A Spanish version of the Lexile framework for reading». Popular Measurement, 25-26.

Treiman, R. \& Kessler, B. (2003). «The role of letter names in the acquisition of literacy». In R. Kail (ed.). Advances in child development and behavior, vol. 31, (pp. 105-135). San Diego, CA: Academic Press.

Vellutino, F.R.; Fletcher, J.M.; Snowling, M.J. \& Scanlon, D.M. (2004). «Specific reading disability (dyslexia): What have we learned in the past four decades?». Journal of Child Psychology and Psychiatry, $45(1), 2-40$.

Villalón, M. (2008). Alfabetización Inicial: Claves de acceso al aprendizaje de la lengua escrita. Santiago de Chile: Ediciones Universidad Católica de Chile.

Wolf, M. \& Katzir-Cohen, T. (2001). «Reading fluency and its intervention». Scientific Studies of Reading, 5, 211-239.

Zimmerman, B.; Padak, N. \& Rasinski, T. (2008). Evidence-based instruction in reading: A professional development guide to phonics. Boston: Pearson Education. 